\title{
Theoretical and experimental analysis of electromagnetic coupling into microwave circuit
}

\author{
Ilham Zerrouk ${ }^{1}$, Mohamed Amellal ${ }^{2}$, Amine Amharech ${ }^{3}$, Mohamed Ramdani $^{4}$, Hassane Kabbaj ${ }^{5}$ \\ ${ }^{1,5}$ Faculty of Sciences and Technologies (FST) Signals, Systems and Components Laboratory (SSCL) Electro-Magnetic \\ Compatibility (EMC) Unit, Morocco \\ ${ }^{2,4}$ Ecole Superieure d'Electronique de l'Ouest (ESEO) Electro-Magnetic Compatibility (EMC) Laboratory, France \\ ${ }^{3}$ National School of Applied Sciences Informatics, Systems, Electrical and Telecommunication Networks Laboratory \\ (ISETNL) Electronics, Signals and Systems (ESS) Unit, Morocco
}

\begin{tabular}{l} 
Article Info \\
\hline Article history: \\
Received Feb 25, 2018 \\
Revised Jun 30, 2018 \\
Accepted Jul 14, 2018 \\
\hline Keywords: \\
EMC \\
FDTD \\
Field-to-line coupling \\
GTEM \\
Immunity measurements \\
MESFET \\
MTL
\end{tabular}

\begin{abstract}
In this paper, our work is devoted to a time domain analysis of field-to-line coupling model. The latter is designed with a uniform microstrip multiconductor transmission line (MTL), connected with a mixed load which can be linear as a resistance, nonlinear like a diode or complex nonlinear as a Metal Semiconductor Field-Effect Transistor (MESFET). The finite difference time-domain technique (FDTD) is used to compute the expression of voltage and current at the line. The primary advantage of this method over many existing methods is that nonlinear terminations may be readily incorporated into the algorithm and the analysis. The numerical predictions using the proposed method show a good agreement with the GHz Transverse Electro Magnetic (GTEM) measurement.
\end{abstract}

Copyright (C) 2019 Institute of Advanced Engineering and Science. All rights reserved.

\section{Corresponding Author:}

Ilham Zerrouk,

Department of Electrical Engineering,

Faculty of Sciences and Technologies,

FSTF BP 2202, Route d'Imouzzer, Fez, Morocco.

Email: ilham.zerrouk@usmba.ac.ma

\section{INTRODUCTION}

Electro-Magnetic Compatibility (EMC) study has become an important step in the realization of electronic systems. Indeed, the technological evolutions require more and more integration of the electronic functions and the electromagnetic coupling effect between external fields and electronic systems. Furthermore, an increased miniaturization of devices shares the same environment. For these reasons, it is fundamental to ensure the proper functioning of the various parts contributing in this environment.

However, electromagnetic illumination, attacking the electronic system, can propagate to the components via the transmission lines, causing the malfunction of the component. In this perspective, the study of the field-line coupling to external electromagnetic excitation allows an accurate prediction of its effect. The radiated immunity of electronic systems is usually measured in a GTEM (Transverse Electromagnetic $\mathrm{GHz}$ ) cell. It illuminates the tested circuit (CUT) with a linearly polarized plane wave widely accepted as a sufficient electromagnetic compatibility tool for radiated susceptibility and radiated emission tests [1].

The analysis of electromagnetic coupling effect has been investigated by various authors and by several techniques [2]-[7]. In this paper, we investigate the analysis of coupling a uniform lossless MTL composed of three conductors and an external plane wave. This MTL is linked to nonlinear terminations as 
the diodes and complex nonlinear loads like the MESFET transistors. The microstrip transmission lines represent an indispensable element for evaluating the performance of Radio Frequency (RF), high-speed digital and microwave systems. In fact, RF and microwave integrated circuits nearly always use microstrip lines for connecting active devices as well as implementing microwave functionality. The MESFET is one of these devices that offer a superior performance, especially in the region of microwave operations and for use within RF amplifiers. Besides, MESFETs extend the advantages of field effect transistors to significantly higher frequencies.

In this paper, we aim to obtain the level of the perturbation generated by an external electromagnetic field in a microwave circuit. The originality of our work is about the passage of the intrinsic large-signal model's transistor to a junction diode. This makes easier the insertion of a mixed load at the far end of the MTL. The method used for the analysis of this structure is the Finite-Difference Time-Domain (FDTD) [8]. It is suitable not only for transient nature problems, but also for the problem of inserting the complex nonlinear components in the MTL. As well as, the used approach has been proven to be the most powerful and accurate tool for dealing with problems involving electromagnetic interactions for many reasons [9]-[11]. It is also interesting to mention that this method is less greedy in memory space, more suitable for the treatment of nonlinear loads and for the resolution of differential equations in the time domain. Hence, the choice of the FDTD method was driven by its simplicity, flexibility, and robustness.

To illustrate the problem and to direct the rest of the study, we will first discuss in section 2 the FDTD algorithm for the calculation of voltages and currents along the line, especially at the input and output with linear termination at both ends of the line. Then, we will give the predictions compared with measurement results of field-to-line coupling model in section 3, followed by conclusion in section 4 .

\section{RESEARCH METHOD}

\subsection{Recursion relations}

Consider a lossless uniform planar MTL in quasi-TEM propagation mode. The MTL equations in the time domain are described by a set of differential equations and the external field is included as a set of distributed series voltage and parallel current sources along the line. These equations are described by [12]:

$$
\begin{aligned}
& \frac{\partial \boldsymbol{V}(z, t)}{\partial z}+\boldsymbol{L} \frac{\partial \boldsymbol{I}(z, t)}{\partial t}=\boldsymbol{V}_{\boldsymbol{F}}(z, t) \\
& \frac{\partial \boldsymbol{I}(z, t)}{\partial z}+\mathbf{C} \frac{\partial \boldsymbol{V}(z, t)}{\partial t}=\boldsymbol{I}_{\boldsymbol{F}}(z, t)
\end{aligned}
$$

with

$$
\mathrm{V}_{\mathrm{F}}(\mathrm{z}, \mathrm{t})=\mathrm{E}_{\mathrm{L}}(\mathrm{z}, \mathrm{t})-\frac{\partial E_{T}(z, t)}{\partial z}
$$

and

$$
I_{\mathrm{F}}(\mathrm{z}, \mathrm{t})=-\mathbf{C} \frac{\partial \boldsymbol{E}_{\boldsymbol{T}}(z, t)}{\partial t}
$$

Where $V(z, t)$ and $I(z, t)$ are $(n \times 1)$ vectors of the total voltages (with respect to the reference conductor) and currents matrices along the MTL. Furthermore, the $V_{F}(z, t)$ and $I_{F}(z, t)$ are $(n \times 1)$ vectors of forcing functions that contain the effects of the incident electromagnetic field. The $(n \times n)$ matrices $L$ and $C$ represent the perunit-length inductance and capacitance respectively, that characterizes the line.

The terms $E_{T}(z, t)$ and $E_{L}(z, t)$ are $(n \times 1)$ vectors having the components of the incident electric field that are transverse to the line and parallel to the line conductors, respectively, with the line conductors removed [2]. The term $\mathrm{E}_{\mathrm{T}}(\mathrm{z}, \mathrm{t})$ is given by:

$$
E_{L}(z, t)=E_{z}^{i, t o t a l}(h, z, t)-E_{z}^{i, t o t a l}(o, z, t)
$$

As already mentioned, in this paper we choose the FDTD method to solve the MTL equations. It is can be used easily to solve several problems either in the transmission line, or the components of the load. In addition, it has a high accuracy for a wide variety of radio frequency (RF) and antenna problems.

As it is widely known, in the FDTD approach, both space and time are divided into discrete segments, the line axis $\mathrm{z}$ is discretized in $\Delta \mathrm{z}$ increments and the time variable $\mathrm{t}$ is discretized in $\Delta \mathrm{t}$ 
increments. The points of current solutions are evaluated at half-time steps and half-spatial positions [13], [14] as shown in Figure 1.

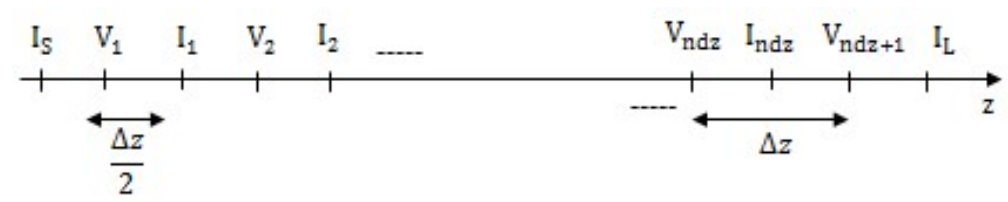

Figure 1. Location of the solution variables on the line

The numerical stability of the FDTD technique is assured when [15], [16].

$$
\Delta t \leq \frac{\Delta z}{v_{p \max }}
$$

where $v_{\text {pmax }}$ is the maximum velocity of the wave propagation.

After the discretization, the induced voltages and currents can be numerically interpreted at integertime steps and integer-spatial positions as:

$$
\begin{aligned}
& \frac{1}{\Delta z}\left(\boldsymbol{V}_{k+1}^{n+1}-\boldsymbol{V}_{k}^{n+1}\right)=-\frac{\boldsymbol{L}}{\Delta t}\left(\boldsymbol{I}_{k}^{n+\frac{3}{2}}-\boldsymbol{I}_{k}^{n+\frac{1}{2}}\right)-\frac{1}{\Delta z}\left(\boldsymbol{E}_{T, \mathrm{k}+1}^{n+1}-\boldsymbol{E}_{T, \mathrm{k}}^{n+1}\right)+\frac{1}{2}\left(\boldsymbol{E}_{L, \mathrm{k}}^{n+\frac{3}{2}}+\boldsymbol{E}_{L, \mathrm{k}}^{n+\frac{1}{2}}\right) \\
& \frac{1}{\Delta z}\left(\boldsymbol{I}_{k}^{n+\frac{1}{2}}-\boldsymbol{I}_{k-1}^{n+\frac{1}{2}}\right)=-\frac{\boldsymbol{C}}{\Delta t}\left(\left(\boldsymbol{V}_{k}^{n+1}-\boldsymbol{V}_{k}^{n}\right)+\left(\boldsymbol{E}_{T, k}^{n+1}-\boldsymbol{E}_{T, k}^{n}\right)\right)
\end{aligned}
$$

Readjusting (8) and (9) gives the voltage equation at the line as follows:

$$
\mathrm{V}_{\mathrm{k}}^{\mathrm{n}+1}=\mathrm{V}_{\mathrm{k}}^{\mathrm{n}}-\left(\mathrm{E}_{\mathrm{T}, \mathrm{k}}^{\mathrm{n}+1}-\mathrm{E}_{\mathrm{T}, \mathrm{k}}^{\mathrm{n}}\right)-\frac{\Delta \mathrm{t}}{\mathrm{C} \Delta \mathrm{z}}\left(\mathrm{I}_{\mathrm{k}}^{\mathrm{n}+\frac{1}{2}}-\mathrm{I}_{\mathrm{k}-1}^{\mathrm{n}+\frac{1}{2}}\right) \quad \text { for } \mathrm{k}=2,3, \ldots, \mathrm{ndz}
$$

Then, the current equation along the line as:

$$
\boldsymbol{I}_{k}^{n+\frac{3}{2}}=\boldsymbol{I}_{k}^{n+\frac{1}{2}}+\frac{\Delta t}{2 L}\left(\boldsymbol{E}_{L, k}^{n+\frac{3}{2}}-\boldsymbol{E}_{L, k}^{n+\frac{1}{2}}\right)-\frac{\Delta t}{L \Delta \mathrm{z}}\left[\left(\boldsymbol{V}_{k+1}^{n+1}-\boldsymbol{V}_{k}^{n+1}\right)+\left(\boldsymbol{E}_{T, k+1}^{n+1}-\boldsymbol{E}_{T, k}^{n+1}\right)\right] \text { for } \mathrm{k}=1,3, \ldots, \mathrm{ndz}
$$

Where we denote

$$
\begin{aligned}
\boldsymbol{V}_{k}^{n} & \equiv \boldsymbol{V}[(k-1) \Delta \mathrm{z}, \mathrm{n} \Delta \mathrm{t}] \\
\boldsymbol{I}_{k}^{n} & \equiv \boldsymbol{I}\left[\left(k-\frac{1}{2}\right) \Delta \mathrm{z}, \mathrm{n} \Delta \mathrm{t}\right] \\
\boldsymbol{E}_{T, k}^{n} & \equiv \boldsymbol{E}_{T}[(k-1) \Delta \mathrm{z}, \mathrm{n} \Delta \mathrm{t}] \\
\boldsymbol{E}_{L, k}^{n} & \equiv \boldsymbol{E}_{L}\left[\left(k-\frac{1}{2}\right) \Delta \mathrm{z}, \mathrm{n} \Delta \mathrm{t}\right]
\end{aligned}
$$

\subsection{Near end voltage}

The quantities of interest are the input and output voltages, which can be found by incorporating boundary conditions. The final updated equation of the line is discretized at the source as:

$$
k=1 ; \quad V_{1}^{n}=V_{s}^{n}-\boldsymbol{R}_{s} \boldsymbol{I}_{s}^{n}
$$


We have then:

$$
\boldsymbol{I}_{s}^{n}=\frac{\boldsymbol{V}_{s}^{n}-\boldsymbol{V}_{1}^{n}}{\boldsymbol{R}_{s}}
$$

The currents $\mathrm{I}_{1}$ and $\mathrm{I}_{\mathrm{s}}$ are separated by $\frac{\Delta z}{2}$ see Figure 1 .

Concluding that:

$$
\boldsymbol{I}_{s}^{n+\frac{1}{2}}=\frac{\boldsymbol{I}_{s}^{n+1}+\boldsymbol{I}_{s}^{n}}{2}
$$

with $R_{S}$ is the source resistance.

The final expression of the near end voltage is then:

$$
\boldsymbol{V}_{1}^{n+1}=\boldsymbol{A}^{-\mathbf{1}}\left[\boldsymbol{B} \cdot \boldsymbol{V}_{1}^{n}-\left(\boldsymbol{E}_{T, 1}^{n+1}-\boldsymbol{E}_{T, 1}^{n}\right)+\frac{\Delta \mathrm{t}}{\boldsymbol{C} \Delta \mathrm{z}}\left[\boldsymbol{R}_{s}^{-1}\left(\boldsymbol{V}_{s}^{n+1}+\boldsymbol{V}_{s}^{n}\right)-2 \boldsymbol{I}_{1}^{n+\frac{1}{2}}\right]\right]
$$

\subsection{Far end voltage}

In this paper, we are interested in the MTL loaded with mixed nonlinear complex loads such as the MESFETs and diode modeled by a nonlinear capacitance in series with a resistance. We are focuses in the nonlinear comportment of the MESFET in common-source configuration, whose drain is connected to a grounded resistor $\mathrm{R}_{\mathrm{L}}$. In Figure 2 shown, we adopt his intrinsic large signal taken from [17], [18]:

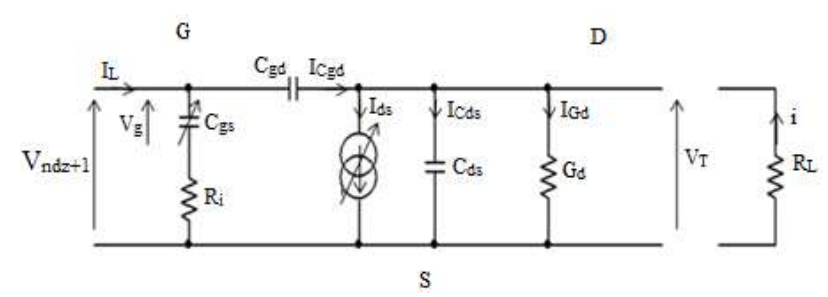

Figure 2. Equivalent circuit of intrinsic large-signal model of MESFET

Where the nonlinearities in this model are represented by gate-source capacitance and drain-source current [19], [20] :

$$
\begin{aligned}
& \boldsymbol{C}_{g s}\left(\boldsymbol{V}_{g}\right)=\frac{\boldsymbol{C}_{g s 0}}{\sqrt{1-\frac{\boldsymbol{V}_{g(t)}}{\boldsymbol{V}_{b}}}} \quad \text { if } V_{g}<V_{b} \\
& \boldsymbol{C}_{\mathrm{gs}}\left(\boldsymbol{V}_{g}\right)=\frac{\boldsymbol{C}_{g s 0}}{\left(1-F_{C}\right)^{\frac{3}{2}}}\left(1-\frac{3}{2} F_{C}+\frac{\boldsymbol{V}_{g}(t)}{2 \boldsymbol{V}_{b}}\right) \text { if } \quad \boldsymbol{V}_{g} \geq\left(\boldsymbol{V}_{b} \cdot F_{C}\right)
\end{aligned}
$$

And

$$
\boldsymbol{I}_{d s}=\left(\boldsymbol{A}_{\mathbf{0}} \boldsymbol{\delta}+\boldsymbol{A}_{\mathbf{1}} \boldsymbol{V}_{g}+\boldsymbol{A}_{\mathbf{2}} \boldsymbol{V}_{g}^{2}+\boldsymbol{A}_{\mathbf{3}} \boldsymbol{V}_{g}^{3}\right) \cdot \tanh \left(\boldsymbol{\alpha} \boldsymbol{V}_{d s}\right)
$$

Where $\mathrm{Vb}$ is the built-in potential of the Schottky gate and $\delta$ is a $(\mathrm{n} \times 1)$ vector of 1 .

Thus a junction diode $\mathrm{Dj}$ in series with a $10 \Omega$ resistor is used for representing the nonlinear load of this structure which is modeled by [21]: 


$$
C_{j}=C_{j 0}\left(1-\frac{V_{d s}}{V_{J}}\right)^{M_{J}}
$$

Where $\mathrm{C}_{\mathrm{j} 0}$ is the zero-bias drain-bulk junction capacitance, $\mathrm{V}_{\mathrm{J}}$ is the junction built-in potential and $\mathrm{M}_{\mathrm{J}}$ is the grading coefficient. Developing current terms of the MESFET yields:

$$
\boldsymbol{I}_{L}(\boldsymbol{t})=\boldsymbol{C}_{g s}\left(\boldsymbol{V}_{g}(t)\right) \frac{d \boldsymbol{V}_{g}(t)}{d t}+\boldsymbol{C}_{g d}\left[\frac{d \boldsymbol{V}_{n d z+1}(t)}{d t}-\frac{d \boldsymbol{V}_{T}(t)}{d t}\right]
$$

In terms of finite difference, $\mathrm{I}_{L}$ can be expressed as:

$$
\begin{aligned}
& \boldsymbol{I}_{L}^{n+1}=\boldsymbol{C}_{g s}\left(\boldsymbol{V}_{g}^{n+1}\right)\left[\frac{\boldsymbol{V}_{g}^{n+1}-\boldsymbol{V}_{g}^{n}}{\Delta t}\right]+\boldsymbol{C}_{g d}\left[\frac{\boldsymbol{V}_{n d z+1}^{n+1}-\boldsymbol{V}_{n d z+1}^{n}}{\Delta t}-\frac{\boldsymbol{V}_{T}^{n+1}-\boldsymbol{V}_{T}^{n}}{\Delta t}\right] \\
& \boldsymbol{I}_{L}^{n}=\boldsymbol{C}_{g s}\left(\boldsymbol{V}_{g}^{n}\right)\left[\frac{\boldsymbol{V}_{g}^{n}-\boldsymbol{V}_{g}^{n-1}}{\Delta t}\right]+\boldsymbol{C}_{g d}\left[\frac{\boldsymbol{V}_{n d z+1}^{n}-\boldsymbol{V}_{n d z+1}^{n-1}}{\Delta t}-\frac{\boldsymbol{V}_{T}^{n}-\boldsymbol{V}_{T}^{n-1}}{\Delta t}\right]
\end{aligned}
$$

The far end voltage is expressed with respect to the current, and is written as follows:

$$
\boldsymbol{V}_{n d z+1}^{n+1}=\boldsymbol{V}_{n d z+1}^{n}-\left(\boldsymbol{E}_{T, n d z+1}^{n+1}-\boldsymbol{E}_{T, n d z+1}^{n}\right)+\frac{2 \Delta \mathrm{t}}{\boldsymbol{C} \Delta \mathrm{z}}\left(\boldsymbol{I}_{n d z}^{n+\frac{1}{2}}-\frac{\boldsymbol{I}_{L}^{n+1}+\boldsymbol{I}_{L}^{n}}{\mathbf{2}}\right)
$$

Equation (21) is obtained by inserting the expressions of $\boldsymbol{I}_{L}^{n}$ and $\boldsymbol{I}_{L}^{n+1}$ into equation (20):

$$
\begin{aligned}
& \boldsymbol{V}_{n d z+1}^{n+1}=\boldsymbol{V}_{n d z+1}^{n}-\frac{\boldsymbol{c}_{g d}}{C \Delta z}\left(\boldsymbol{V}_{n d z+1}^{n+1}-\boldsymbol{V}_{n d z+1}^{n-1}\right)-\frac{\boldsymbol{c}_{g s}}{C \Delta z}\left[\left(\boldsymbol{V}_{g}^{n+1}-\boldsymbol{V}_{g}^{n}\right)+\left(\boldsymbol{V}_{g}^{n}-\boldsymbol{V}_{g}^{n-1}\right)\right]+ \\
& \frac{\boldsymbol{c}_{g d}}{\boldsymbol{C} \Delta z}\left(\boldsymbol{V}_{T}^{n+1}-\boldsymbol{V}_{T}^{n-1}\right)+\frac{2 \Delta t}{c \Delta z} \boldsymbol{I}_{n d z}^{n+\frac{1}{2}}-\left(\boldsymbol{E}_{T, n d z+1}^{n+1}-\boldsymbol{E}_{T, n d z+1}^{n}\right)
\end{aligned}
$$

According to the model in Figure 2 we have:

$$
\boldsymbol{V}_{n d z+1}^{n+1}=\boldsymbol{V}_{g}^{n+1}+\boldsymbol{R}_{i} \boldsymbol{C}_{g s}\left(\boldsymbol{V}_{g}^{n+1}\right)\left[\frac{\boldsymbol{V}_{g}^{n+1}-\boldsymbol{V}_{g}^{n}}{\Delta \mathrm{t}}\right]
$$

Replacing $\boldsymbol{V}_{n d z+1}$ of equation (22) in (21) gives:

$$
\begin{aligned}
& \boldsymbol{V}_{T}^{n+1}=\frac{\boldsymbol{C \Delta z}}{\boldsymbol{C}_{g d}}\left[\boldsymbol{V}_{g}^{n+1}+\frac{\boldsymbol{R}_{i} \boldsymbol{c}_{g s}}{\Delta \mathrm{t}}\left(\boldsymbol{V}_{g}^{n+1}-\boldsymbol{V}_{g}^{n}\right)-\boldsymbol{V}_{n d z+1}^{n}+\left(\boldsymbol{E}_{T, n d z+1}^{n+1}-\boldsymbol{E}_{T, n d z+1}^{n}\right)\right]+ \\
& \frac{\boldsymbol{C}_{g s}}{\boldsymbol{C}_{g d}}\left[\left(\boldsymbol{V}_{g}^{n+1}-\boldsymbol{V}_{g}^{n}\right)+\left(\boldsymbol{V}_{g}^{n}-\boldsymbol{V}_{g}^{n-1}\right)\right]+\boldsymbol{V}_{g}^{n+1}+\frac{\boldsymbol{R}_{i} \boldsymbol{C}_{g s}}{\Delta \mathrm{t}}\left(\boldsymbol{V}_{g}^{n+1}-\boldsymbol{V}_{g}^{n}\right)+\boldsymbol{V}_{T}^{n-1}-\boldsymbol{V}_{n d z+1}^{n-1}- \\
& \frac{2 \Delta \mathrm{t}}{\boldsymbol{C}_{g d}} \boldsymbol{I}_{n d z}^{n-\frac{1}{2}}
\end{aligned}
$$

According to the scheme in Figure 2 we have:

$$
\boldsymbol{V}_{T}^{n+1}=-\boldsymbol{R}_{L} i^{n+1}=-\boldsymbol{R}_{L}\left(\boldsymbol{I}_{C d s}^{n+1}-\boldsymbol{I}_{C g d}^{n+1}+\boldsymbol{I}_{G d}^{n+1}+\boldsymbol{I}_{d s}^{n+1}\right)
$$

with

$$
\begin{aligned}
\boldsymbol{I}_{C d s}^{n+1} & =\frac{\boldsymbol{C}_{\boldsymbol{d s}}}{\Delta t}\left(\boldsymbol{V}_{T}^{n+1}-\boldsymbol{V}_{T}^{n}\right) \\
\boldsymbol{I}_{C g d}^{n+1} & =\frac{\boldsymbol{C}_{g d}}{\Delta t}\left(\boldsymbol{V}_{n d z+1}^{n+1}-\boldsymbol{V}_{n d z+1}^{n}-\boldsymbol{V}_{T}^{n+1}+\boldsymbol{V}_{T}^{n}\right) \\
\boldsymbol{I}_{G d}^{n+1} & =\boldsymbol{G}_{\boldsymbol{d}} \boldsymbol{V}_{T}^{n+1}
\end{aligned}
$$


and

$$
\boldsymbol{I}_{d s}^{n+1}=\left(\boldsymbol{A}_{\mathbf{0}} \delta+\boldsymbol{A}_{\mathbf{1}} \boldsymbol{V}_{g}^{n+1}+\boldsymbol{A}_{\mathbf{2}}\left(\boldsymbol{V}_{g}^{n+1}\right)^{2}+\boldsymbol{A}_{\mathbf{3}}\left(\boldsymbol{V}_{g}^{n+1}\right)^{3}\right) \cdot \tanh \left(\boldsymbol{\alpha} \boldsymbol{V}_{T}^{n+1}\right)
$$

The only unknown term in all these equations is $\boldsymbol{V}_{g}^{n+1}$, which can be solved by using the NewtonRaphson method as shown:

$$
\boldsymbol{H} \equiv-\boldsymbol{V}_{g}^{n+1}+f\left(\boldsymbol{V}_{g}^{n+1}\right)=0
$$

Where $\mathrm{f}$ is a term of $\mathrm{V}_{\mathrm{g}}^{\mathrm{n}+1}$ and where the nonlinear components are collected. The matrix $\mathrm{V}_{\mathrm{g}}^{\mathrm{n}+1}$ is found by solving the following equation:

$$
\left(\boldsymbol{V}_{g}^{n+1}\right)^{m+1}=\left(\boldsymbol{V}_{g}^{n+1}\right)^{m}-\left(\boldsymbol{J}_{\boldsymbol{H}}\right)^{-1} \boldsymbol{H}
$$

where:

$$
\boldsymbol{J}_{\boldsymbol{H}}=\frac{d \boldsymbol{H}}{d \boldsymbol{V}_{g}} \mid \boldsymbol{V}_{g}=\left(\boldsymbol{V}_{g}\right)^{m}
$$

Once $\boldsymbol{V}_{g}^{n+1}$ found, we can determine $\boldsymbol{V}_{T}^{n+1}$ and $\boldsymbol{V}_{n d z+1}^{n+1}$

\section{NUMERICAL AND MEASUREMENT RESULTS}

The transient response of the printed circuit board (PCB) multiconductor transmission lines discussed above will be evaluated by FDTD approach and GTEM measurements. Our case study is a MTL terminated at the near end of $50 \Omega$ resistors $\left(R_{S 1}=R_{S 2}=R_{S 3}=50 \Omega\right)$. Furthermore, this structure is connected with the arbitrary nonlinear loads as MESFETs, diode and resistances at the far end. In this case the line is illuminated by an incident electromagnetic field [22], whose incident, azimuth and polarization angles are respectively: $\theta_{p}=90^{\circ}, \theta_{E}=90^{\circ}, \varphi_{p}=-90^{\circ}$.

As an example of results obtained by the presented approach and GTEM cell, we choose three lossless conductors transmission line see Figure 3 . The track width of every conductor is $\mathrm{w}=3 \mathrm{~mm}$ are separated by $1 \mathrm{~cm}$ and placed on one side of a FR4 substrate having $\varepsilon_{r}=4.5$, thickness $\mathrm{h}=1.64 \mathrm{~mm}$, and the total line length is $\ell=3 \mathrm{~cm}$.

Knowing these parameters, the per-unit-length inductance and capacitance matrices of the circuit are computed according to the numerical method giving [23].

$$
\begin{aligned}
\boldsymbol{L} & =\left(\begin{array}{lll}
0.313116 & 0.0063895 & 0.0015995 \\
0.0063895 & 0.313111 & 0.0063894 \\
0.0015995 & 0.0063894 & 0.313116
\end{array}\right) n H / m \\
\boldsymbol{C} & =\left(\begin{array}{lll}
120.276 & -0.45951 & -0.10285 \\
-0.45951 & 120.278 & -0.45951 \\
-0.10285 & -0.45951 & 120.276
\end{array}\right) p F / m
\end{aligned}
$$

The Figure 3 displays the scheme of our circuit under test. As displayed in the scheme, the nonlinear complex termination is represented by a MESFET modeled by its intrinsic large-signal model and terminated of $50 \Omega$. The measurements were performed in the EMC laboratory at the ESEO of Angers-France. As illumination for our circuit, the GTEM cell was chosen as the plane wave source with an input power Pin $=40 \mathrm{dBm}$ and a frequency swept from $50 \mathrm{MHz}$ to $4 \mathrm{GHz}$. 


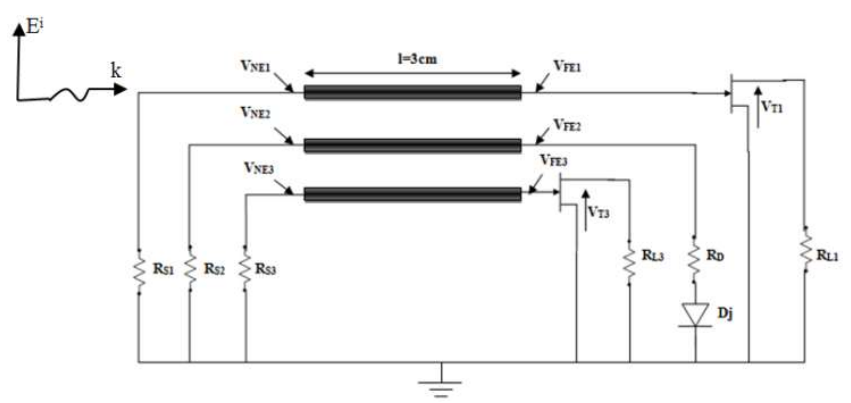

Figure 3. Circuit under test: Microstrip MTL connected witch MESFETs and diode

The GTEM cell can be treated a characteristically terminated $50 \Omega$ waveguide with an input connector. The Figure 4 shown display the whole equipment, which allows us to carry out the immunity measurement of our circuit. RF generator for immunity measurements of ESEO-EMC Laboratory as shown in Figure 5.
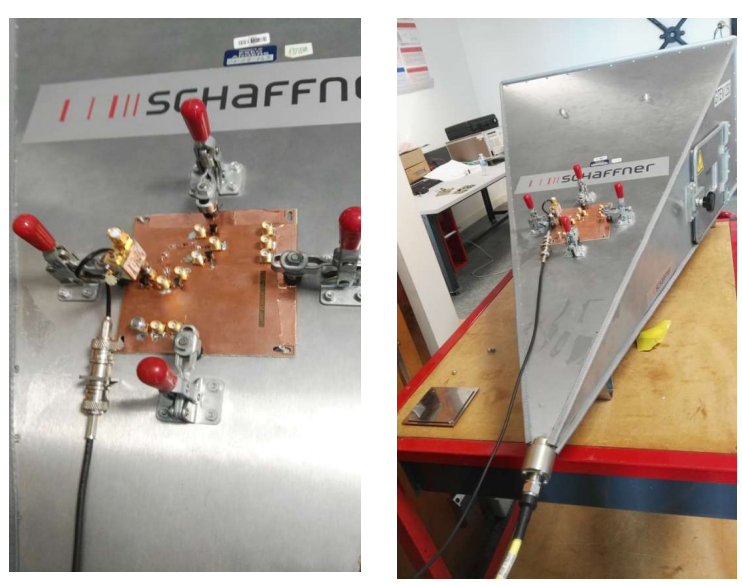

Figure 4. Circuit under test in the GTEM
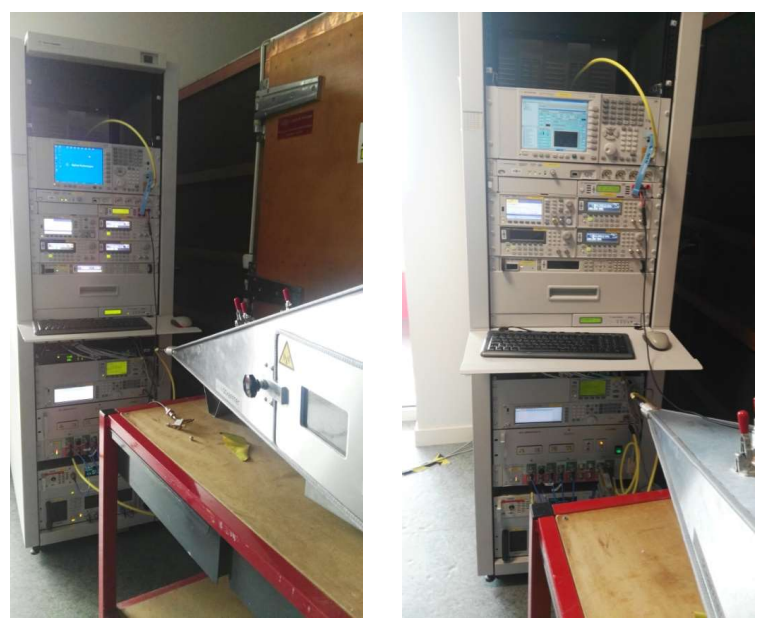

Figure 5. RF generator for immunity measurements of ESEO-EMC Laboratory

As well known the results obtained by the FDTD algorithm are in the time domain, contrariwise to those obtained with the GTEM cell (frequency), that's why we used the fast Fourier transform algorithm 
(FFT) to compare both results.We mention that our choice of conductors is arbitrary. The Figure 6 shown the numerical and measurement results of the near ends and output transistor voltages:

In this section, it is explained the results of research and at the same time is given the comprehensive discussion. Results can be presented in figures, graphs, tables and others that make the reader understand easily [2], [5]. The discussion can be made in several sub-chapters.

The Figure 6(a) illustrates the voltages induced at the input of the first and third conductors. As we can notice the voltage reaches its maximum at the value of the rise time of the external wave settled to $1 \mathrm{~ns}$. Besides, the obtained results are in good agreement with those measured by GTEM cell as shown in Figure 6(b) The results, displayed in Figure 7, present the effect of the external plane wave to the MESFET transitor. We observe that the level of the perturbation is nearly $15 \mathrm{mV}$. However, this value can deteriorate the transistor gain and causing a malfuction not only for the component, but also for the microwave circuit.

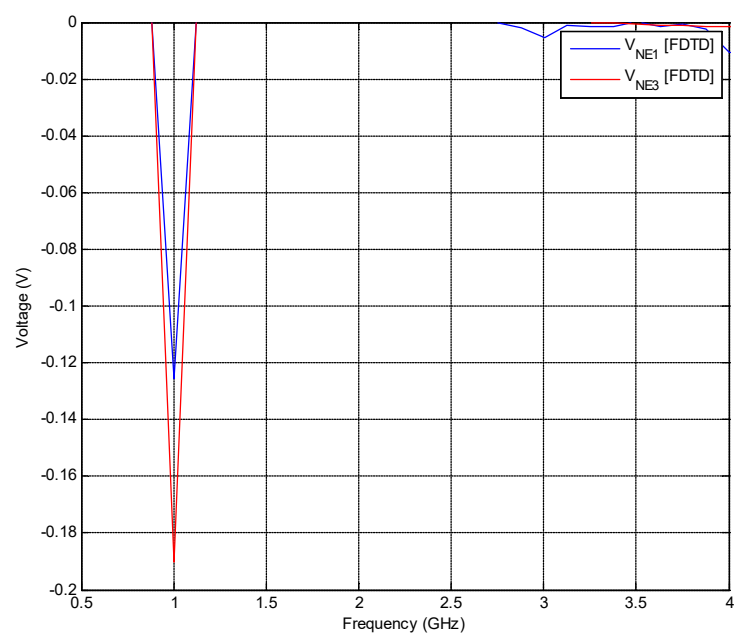

(a)

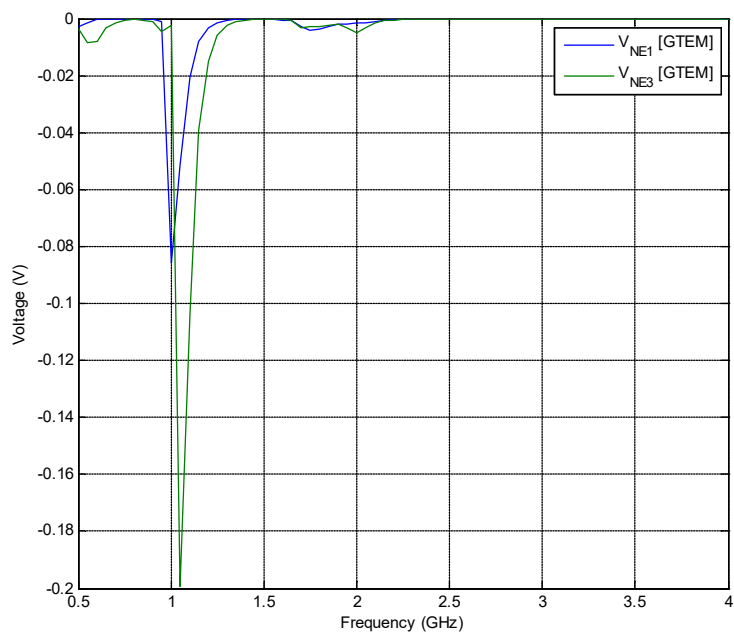

(b)

Figure 6. Near end voltage: (a) FDTD approach; (b) GTEM measurements

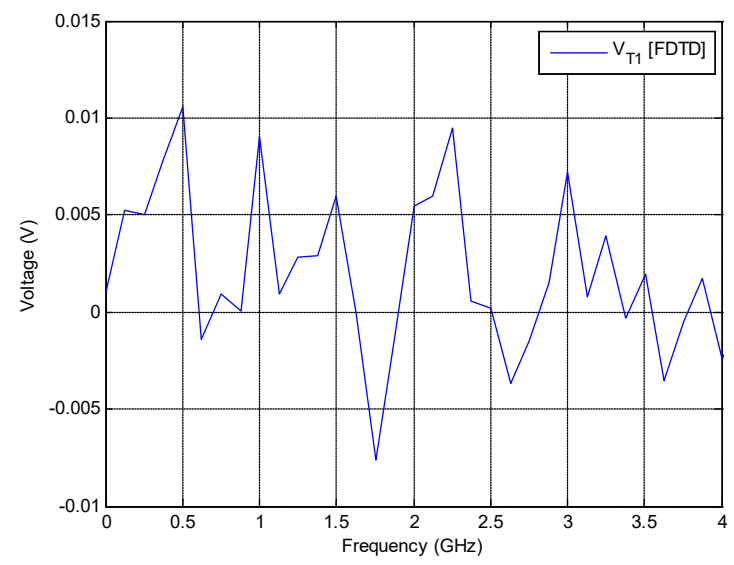

(a)

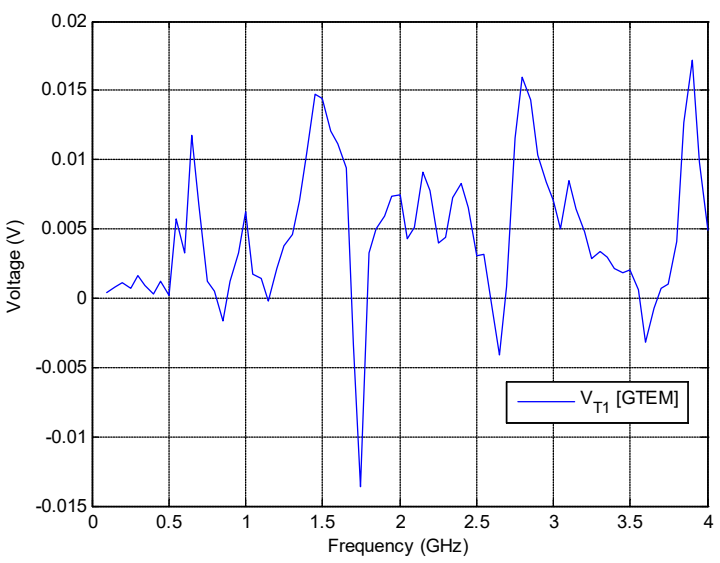

(b)

Figure 7. Transistor's output voltage of the $1^{\text {st }}$ conductor: (a) FDTD approach; (b) GTEM measurements

\section{CONCLUSION}

This paper presents a technique to conduct a time-domain analysis of field-to-line coupling. The proposed time-domain code is principally based on discretizing MTL equations by means of FDTD 
approach along with the use of some numerical functions, to determine the electromagnetic coupling effect at both ends of MTL.

In the order to readily integrate a mixed complex nonlinear load at the end of MTL, we have implemented a code to switch the intrinsic large signal model's transistor to a junction diode. The code has been verified and validated by confronting the obtained results with those carried out with GTEM cell (IEC 61967-2/18GHz) realized in ESEO-EMC laboratory, which has been widely accepted as a sufficient tool in electromagnetic compatibility for both radiated susceptibility tests and radiated emission tests.

After several simulations, the developed approach turned out to be simple as well as a powerful numerical technique. Arbitrary nonlinear complex loads can easily be dealt without major constraints. As a matter of fact, we treated a lossless multiconductor microstrip line terminated with MESFETs transistor and a junction diode.

Predictions have been computed for the simulation parameters $\Delta \mathrm{z}=1 \mathrm{~mm}$ and $\Delta \mathrm{t}=4 \mathrm{ps}$. These results are compared with those measured by GTEM cell with a frequency swept from 50MHz to $4 \mathrm{GHz}$ and an input power Pin $=40 \mathrm{dBm}$. Finally, identical results have been achieved that prove the simplicity and efficiency of the new time domain technique as well as a good choice of time and space discretization to satisfy the stability condition in the method.

\section{REFERENCES}

[1] M. Amellal, "Electromagnetic Immunity Modeling of Components for the Obsolescence Management of Systems and Electronic Modules," Theses, INSA de Rennes, https://tel.archives-ouvertes.fr/tel-01333563, 2015.

[2] G.S. Shinh, N. M. Nakhla, R. Achar, et al., "Fast transient Analysis of Incident Field Coupling to Multiconductor Transmission Lines," IEEE Trans Electromagn Compat, vol. 48, pp. 57-73, 2006.

[3] G. Antonini, "A Spectral Formulation for the Transient Analysis Of Plane-Wave Coupling To Multiconductor Transmission Lines," IEEE Trans Electromagn Compat, vol. 51, pp. 792-804, 2009.

[4] I. Zerrouk, H. Kabbaj, A. Amharech, "Transient Response of Multiconductor Transmission Line, Terminated in Complex Nonlinear Loads and illuminated by an External Electromagnetic Field," vol. 6, pp. 931-938,Nov. 2015.

[5] Y. P. Sun, S. Zhang, X.H. An and H. P. Sun, "Characteristics of Electromagnetic Pulse Coupling into Annular Apertures," Telecomunication Computing Electronics and Control (TELKOMNIKA), vol. 11, pp. 6848-6854, 2013.

[6] Y. P. Sun, L. Sun, L. Qu and X. H. An, "Characteristics of Electromagnetic with A wire Through Enclosure,", Indones. J. Electr. Eng., vol. 12, pp. 1438-1445, 2014

[7] Y. P. Sun, L. Qu and L. Sun,"Analysis on Electromagnetic Interference with Different Polarization of Electric Field," Indonesian Journal of. Electrical. Engineering and Computer Science (IJEECS)., vol. 12, pp. 3684-3689, 2014.

[8] K. S. Kunz, R. J. Luebbers, "The Finite Difference Time Domain Method for Electromagnetics," CRC press, 1993.

[9] G. Ngendakumna, N. El Ouazzani, "Simulation of Ultra Wideband Miscrostrip Antenna Based on Laguerre-FiniteDifference Time-Domain (LFDTD) Algorithm," Int J Comput Sci Netw Secur IJCSNS, pp. 13-33, 2013.

[10] D. M. Sullivan, "Electromagnetic Simulation Using the FDTD Method," John Wiley \& Sons, 2013.

[11] H. Harkat, H. D. Bennani, "Ground Penetrating Radar Imaging for Buried Cavities in a Dispersive Medium: Profile Reconstruction Using a Modified Hough Transform Approach and a Time-Frequency Analysis," Int. J. Commun. Antenna Propag. IRECAP, pp. 78-92, 2015.

[12] C. R. Paul, "Analysis of Multiconductor Transmission Lines," John Wiley \& Sons, 2008.

[13] K. Afrooz, A. Abdipour, "Efficient Method for Time-Domain Analysis of Lossy Nonuniform Multiconductor Transmission Line Driven by a Modulated Signal Using FDTD Technique," IEEE Trans Electromagn Compat, pp. 482-494, 2012.

[14] J. SONG, F. Yuan, Z. Ding, et al., "Transient Analysis of Transmission Lines Based Unconditionally Stable FDTD Method [J]," J Jilin Univ Eng Technol, 5: 052, 2010.

[15] K. Li, M. Tassoudji, R. Shin, et al., "Simulation of Electromagnetic Radiation and Scattering Using A Finite Difference-Time Domain Technique," Comput Appl Eng Educ, pp. 45-63, 1992.

[16] J. A. Roden, C. R. Paul, W. T. Smith, et al., "Finite-difference, Tme-Domain Analysis Of Lossy Transmission Lines," IEEE Trans. Electromagn. Compat., vol. 38, pp. 15-24, 1996.

[17] I. Maio, F. G. Canavero, "Analysis of Crosstalk and Field Coupling To Lossy MTLs in a SPICE Environment," vol. 38, pp. 221-229, 1996.

[18] E. K. Chien, B. Houshmand, and T. Itoh, "Full-wave Analysis of Packaged Microwave Circuits with Active and Nonlinear Devices: an FDTD Approach," IEEE Trans. On Microwave Theory and Techniques, vol. 45, pp. 819826, 1997.

[19] G. Alsharahi, A. Faize, A. M. Mohamed Mostapha, and A. Driouach, "2D FDTD Simulation to Study Response of GPR Signals in Homogeneous and Inhomogeneous Mediums," Int. J. Commun. Antenna Propag. IRECAP, 2010.

[20] J. W. Bandler et al., "Efficient Large-Signal FET Parameter Extraction Using Harmonics," IEEE Trans. on Microwave Theory Tech., vol. 37, pp. 2099-2108, 1989.

[21] S. Lee, "Empirical Nonlinear Modeling for RF MOSFETs," pp. 182-189, 2004.

[22] H. Kabbaj, "Temporal Study of the Interaction Between An External Electromagnetic Wave and a Transmission Line with Complex Monlinear Load," ANN. TELECOMM., vol. 57, pp. 38-58, 2002.

[23] C. R. Paul, "Analysis of Multiconductor Transmission Lines," First edition, John Wiley \& Sons, 1994.

Theoretical and experimental analysis of electromagnetic coupling into microwave circuit (Ilham Zerrouk) 